\title{
On the Stability of Linear Repetitive Processes Described by a Delay-Difference Equation
}

\author{
E. Rogers and D. H. Owens
}

\begin{abstract}
This paper considers linear repetitive processes which are a distinct class of two-dimensional linear systems of both physical and systems theoretic interest. Their essential unique feature is a series of sweeps, termed passes, through a set of dynamics defined over a finite and fixed duration known as the pass length. The result can be oscillations in the output sequence of pass profiles which increase in amplitude in the pass-to-pass direction. This cannot be controlled by existing techniques and instead control must be based on a suitably defined stability theory. In the literature to date, the development of such a theory has been attempted from two different starting points, and in this paper, we critically compare these for dynamics defined by a delay-difference equation.
\end{abstract}

Index Terms-Two-dimensional (2-D) systems, repetitive processes, stability.

\section{INTRODUCTION}

$\mathbf{T}$ HE DYNAMICS of repetitive (also termed multipass in the early literature) processes can be illustrated by considering machining operations, where the material or workpiece involved is processed by a sequence of passes of the processing tool. Assuming the pass length $\alpha<\infty$ to be constant, the output vector, termed the pass profile $y_{k}(t) 0 \leq t \leq \alpha$ ( $t$ being the independent spatial or temporal variable), generated on pass $k$, acts as a forcing function on the next pass and hence contributes to the dynamics of the new pass profile $y_{k+1}(t), 0 \leq t \leq \alpha$, and $k \geq 0$. This, in turn, leads to the unique control problem for these processes in that the output sequence of pass profiles generated can contain oscillations that increase in amplitude in the pass-to-pass direction.

Physical examples of repetitive processes include long-wall coal cutting and metal rolling operations-see, for example, [1]. Also, in recent years, applications have arisen where adopting a repetitive process setting for analysis has distinct advantages over alternatives. Examples of these so-called algorithmic applications include classes of iterative learning control (ILC) schemes [4] and iterative algorithms for solving nonlinear dynamic optimal control problems based on the maximum principle [5]. In the case of ILC for the linear dynamics case, the stability theory for differential and discrete linear repetitive processes is the essential basis for a rigorous

Manuscript received April 22, 2003; revised August 13, 2003. This paper was recommended by Associate Editor R. Dogaru.

E. Rogers is with the School of Electronics and Computer Science, University of Southampton, Southampton SO17 1BJ, U.K. (e-mail: etar@ecs.soton.ac.uk).

D. H. Owens is with the Department of Automatic Control and Systems Engineering, University of Sheffield, Sheffield S1 3JD, U.K.

Digital Object Identifier 10.1109/TCSII.2004.829566 stability/convergence analysis of a powerful class of such algorithms.

Attempts to control these processes using standard, termed one-dimensional (1-D) here, systems theory/algorithms fail (except in a few very restrictive special cases) precisely because such an approach ignores their inherent two-dimensional (2-D) systems structure, i.e., information propagation occurs from pass-to-pass and along a given pass, and also the effects of resetting the pass initial conditions before the start of each new pass. Moreover, the fact that information propagation in one of the two independent directions (along the pass) only occurs over a finite duration is the core reason why they are distinct from other classes of 2-D systems, such as those described by the well known and extensively studied Roesser and Fornasini-Marchesini state-space models, see, for example, the relevant cited references in [3].

The first attempt at stability analysis for these processes was in the setting of the long-wall coal cutting and metal rolling examples [2] and was based on first converting the (assumed) linear dynamics into those of an equivalent infinite-length single-pass process and then applying 1-D linear stability theory and tests. An alternative stability theory is that based on an abstract model of the dynamics in a Banach space setting [6], which includes all processes with linear dynamics and a constant pass length as special cases and, crucially, takes explicit account of both the finite pass length and the resetting of the initial conditions before the start of each new pass.

The stability analysis of [2] is, however, easier to apply in certain cases of practical interest (such as linear single-input single-output dynamics which arise as approximate models in the long-wall coal cutting and metal rolling applications areas) and hence, there is interest in establishing if, and under what conditions, these two approaches to stability are equivalent. Here, we answer this question for the case of repetitive processes described by a delay-difference equation.

\section{Process Modeling}

With $\alpha<\infty$ denoting the pass length, the following [6] is the abstract model for linear repetitive processes.

Definition 1: A linear repetitive process of constant pass length $\alpha$ consists of a Banach space $E_{\alpha}$, a linear subspace $W_{\alpha}$ of $E_{\alpha}$, and a bounded linear operator $L_{\alpha}$ mapping $E_{\alpha}$ into itself. The process dynamics are described by linear recursion relations of the form

$$
y_{k+1}=L_{\alpha} y_{k}+b_{k+1}, \quad k \geq 0
$$

where $y_{k} \in E_{\alpha}$ is the pass profile on pass $k$ and $b_{k+1} \in W_{\alpha}$. Here, the term $L_{\alpha} y_{k}$ represents the contribution of pass $k$ to pass 
$k+1$ and $b_{k+1}$ represents known initial conditions, disturbances and control input effects which enter on pass $k+1$. We denote this abstract model by $S$ here.

The particular subject of this paper is single-input singleoutput linear repetitive processes which can be modeled by the following delay-difference equation:

$$
\begin{aligned}
y_{k+1}(t)= & -k_{1} y_{k+1}(t-X)+k_{2} y_{k}(t)+k_{3} R_{k+1}(t), \\
& 0 \leq t \leq \alpha ; \quad k \geq 0 .
\end{aligned}
$$

Here, $k_{1}, k_{2}$, and $k_{3}$ are real constants, $X>0$ is a transport delay or lag on the current pass dynamics, and $R_{k+1}(t)$ is the current pass input open loop or the current pass reference vector closed loop under the action of feedback control. Note also that the delay term $(X)$ is an essential feature in some physical examples such as long-wall coal cutting (see [1], [2]). In particular, in the model of the long-wall coal cutting dynamics, $y_{k+1}(t)$ is the coal thickness above some datum plane which can only be measured by placing a sensor some distance $(X)$ behind the cutting drum and to ignore this deletes a critical feature of the process dynamics from the model to be used for onward analysis.

To complete the process description, it is necessary to specify the boundary conditions, i.e., the initial condition on each pass the initial pass profile. In the case of the first of these, the simplest possible choice is

$$
y_{k}(t)=0, \quad-X \leq t \leq 0 ; \quad k \geq 1
$$

and for the second $y_{0}(t)=f(t)$, where $f(t)$ is a known function of $t$ over $0 \leq t \leq \alpha$. It is also possible that the initial condition on each pass is an explicit function of the previous pass profile. One way of representing this is

$$
y_{k+1}(t)=c y_{k}(\alpha-|t|), \quad-X \leq t \leq 0
$$

where $c$ is a nonzero real scalar. (This is one case of explicit interaction between pass profiles and pass initial conditions which is another distinct feature of repetitive processes.)

To write the model of (1) and (2) as a special case of the abstract model $S$ of Definition 1, let $E_{\alpha}=W_{\alpha}$ be the vector space of continuous functions over $[0, \alpha]$ on satisfying the initial condition $y(0)=0$ with norm $\|y\|=\max _{0 \leq t \leq \alpha}|y(t)|$, Then, the operator $L_{\alpha}$ is defined by writing $y_{1}=L_{\alpha} y_{0}$ as

$$
\begin{aligned}
& y_{1}(t)=-k_{1} y_{1}(t-X)+k_{2} y_{0}(t), \quad 0 \leq t \leq \alpha \\
& y_{1}(t)=0, \quad-X \leq t \leq 0 .
\end{aligned}
$$

The construction of $L_{\alpha}$ in the case of (3) is a straightforward extension of the previous case and hence the details are omitted here. Also, the basis for the stability analysis of the processes considered here using the approach of [2] is introduced in the context of the later in this paper.

\section{STABILITY ANALYSIS}

Given the unique control problem, i.e., oscillations that increase in amplitude from pass-to-pass, a natural definition of stability is to ask that bounded sequences of disturbances produce bounded sequences of pass profiles. The following is the formal definition of asymptotic stability of linear repetitive processes described by $S$, where it is also required that this property is retained in the presence of "small" additive perturbations to the model of the process dynamics and $\|\cdot\|$ is used to denote the norm on $E_{\alpha}$.

Definition 2: The linear repetitive process $S$ is said to be asymptotically stable [6] if there exists a real scalar $\delta>0$ such that, given any initial profile $y_{0}$ and any disturbance sequence $\left\{b_{k}\right\}_{k \geq 1} \in W_{\alpha}$ bounded in norm, i.e., $\left\|b_{k}\right\| \leq c_{1}$, for some constant $c_{1} \geq 0$ and $\forall k \geq 1$, the output sequence generated by the perturbed process

$$
y_{k+1}=\left(L_{\alpha}+\gamma\right) y_{k}+b_{k+1}, \quad k \geq 0
$$

is bounded in norm whenever $\|\gamma\| \leq \delta$.

This definition is easily shown to be equivalent to the requirement that there exist finite real scalars $M_{\alpha}>0$ and $\lambda_{\alpha} \in(0,1)$ such that

$$
\left\|L_{\alpha}^{k}\right\| \leq M_{\alpha} \lambda_{\alpha}^{k}, \quad k \geq 0
$$

(where $\|\cdot\|$ is also used to denote the induced operator norm).

Following [6], it can be shown that this last property holds if, and only if

$$
r\left(L_{\alpha}\right)<1
$$

where $r(\cdot)$ denotes the spectral radius of its argument. Hence, for any special case of $S$, the essential task in checking this last condition is to find the spectral values of the associated $L_{\alpha}$.

A standard approach to computing the spectral values of a bounded linear operator mapping a Banach space into itself is to consider the equation

$$
\left(\eta I-L_{\alpha}\right) y=x_{0}
$$

and construct necessary and sufficient conditions on the complex scalar $\eta$ to ensure that a solution exists for all $x_{0} \in E_{\alpha}$ and which is bounded in the sense that $\|y\| \leq K_{0}\left\|x_{0}\right\|$ for some real scalar $K_{0}>0$ and for all $x_{0} \in E_{\alpha}$. Suppose also that $\mathcal{A}$ denotes the set of values of $\eta$ for which this last property is not true. Then, by definition (see, for example, the relevant references in [6] for the background) the spectral radius of $L_{\alpha}$ is the supremum over the moduli of the elements in this set.

The following result now gives the necessary and sufficient condition for the case of processes described by (1) and (2).

Theorem 1: The linear repetitive process $S$ generated by a process modeled by (1) and (2) is asymptotically stable if, and only if

$$
\left|k_{2}\right|<1
$$

Proof: In this case, (6) can be written as

$$
\eta y(t)-w(t)=x_{0}(t)
$$

where

$$
\begin{aligned}
& w(t)=-k_{1} w(t-X)+k_{2} y(t) \\
& w(t)=0, \quad-X \leq t \leq 0 .
\end{aligned}
$$

Since $X>0$ by assumption, it is possible to choose an integer $n \geq 1$ such that $(n-1) X<\alpha \leq n X$. Also, after some 
manipulation, it is possible to write this last expression in the form

$$
\begin{aligned}
& \eta\left[\begin{array}{c}
y(t) \\
y(t+X) \\
\vdots \\
\vdots \\
y(t+(n-1) X)
\end{array}\right]-\left[\begin{array}{c}
w(t) \\
w(t+X) \\
\vdots \\
\vdots \\
w(t+(n-1) X)
\end{array}\right] \\
& =\left[\begin{array}{c}
x_{0}(t) \\
x_{0}(t+X) \\
\vdots \\
\vdots \\
x_{0}(t+(n-1) X)
\end{array}\right] \\
& {\left[\begin{array}{ccccc}
1 & 0 & \ldots & \ldots & 0 \\
k_{1} & 1 & \ldots & \ldots & \vdots \\
0 & k_{1} & \ldots & \ldots & \vdots \\
0 & & & 1 & 0 \\
0 & \ldots & 0 & k_{1} & 1
\end{array}\right]\left[\begin{array}{c}
w(t) \\
w(t+X) \\
\vdots \\
\vdots \\
w(t+(n-1) X)
\end{array}\right]} \\
& =k_{2}\left[\begin{array}{c}
y(t) \\
y(t+X) \\
\vdots \\
\vdots \\
y(t+(n-1) X)
\end{array}\right], \quad 0 \leq t \leq X
\end{aligned}
$$

or, on eliminating the $w$ variables

$$
\left(z I_{n}-K\right)\left[\begin{array}{c}
y(t) \\
y(t+X) \\
\vdots \\
\vdots \\
y(t+(n-1) X)
\end{array}\right]=\left[\begin{array}{c}
x_{0}(t) \\
x_{0}(t+X) \\
\vdots \\
\vdots \\
x_{0}(t+(n-1) X)
\end{array}\right],
$$

where the $n \times n$ matrix $K$ is a lower triangular with the structure

$$
\begin{aligned}
K_{i, i} & =k_{2}, & & 1 \leq i \leq n \\
K_{i+1, j+1} & =K_{i, j}, & & 1 \leq i ; \quad j \leq n-1 .
\end{aligned}
$$

Taking $\eta \neq k_{2}$, it is clear that (7) has a solution at each point $t \in$ $[0, X]$ and it is easily shown that $y(0)=0$ and that the function $y(t)$ is continuous over the pass length $0 \leq t \leq \alpha$. Consider also (without loss of generality) the norm $\|x\|=\max _{1<j \leq n}\left|x_{j}\right|$ in $\mathbb{R}^{n}$. Then, it is clear from (7) that

$$
\|y\| \leq\left\|\left(\eta I_{n}-K\right)^{-1}\right\|\left\|x_{0}\right\| .
$$

Hence, the only candidate for a spectral value of $L_{\alpha}$ is $\eta=k_{2}$.

In the case, when $\eta=k_{2}$, the matrix $\eta I-K$ is singular and it is routine to verify that we can construct $x_{0}$ such that (7) has no solution. Hence, that the set $\mathcal{A}$ of spectral values for this operator is given by $\mathcal{A}=\left\{k_{2}\right\}$ and, therefore, $r\left(L_{\alpha}\right)=\left|k_{2}\right|$. The proof is now completed using (5).

In the case of processes described by (1) and (3), the same steps as in the proof of the last result apply but the matrix $K$ is no longer lower triangular and hence the necessary and sufficient condition for asymptotic stability can only be stated as $r(K)<$ 1 . This point is critical in the sense that it shows that asymptotic stability of these processes is critically dependent on the form of the initial conditions assumed at the start of each new pass. The implications of this fact are considered in detail later in this section.

For the general model $S$ of Definition 1, (5) gives the necessary and sufficient condition for asymptotic stability but little or no information concerning transient behavior where here there are two parts to be considered. These are pass-to-pass and along the pass, respectively.

In the case of the former, suppose that $S$ is asymptotically stable and the disturbance sequence applied $\left\{b_{k}\right\}_{k \geq 1}$ converges strongly to a disturbance $b_{\infty}$. Then the strong limit

$$
y_{\infty}:=\lim _{k \rightarrow \infty} y_{k}
$$

is termed the limit profile corresponding to this disturbance sequence, i.e., after a "large" number of passes the dynamics converge in the pass-to-pass direction. Following [6], we have that under asymptotic stability, the resulting limit profile $y_{\infty}$ is the unique solution of the linear equation

$$
y_{\infty}=L_{\alpha} y_{\infty}+b_{\infty} .
$$

For the case of processes described by (1) and (2), the resulting limit profile under asymptotic stability is the unique solution (where $R_{\infty}$ is the strong limit of the sequence $\left\{R_{k}\right\}_{k \geq 1}$ ) of

$$
\begin{aligned}
y_{\infty}(t)= & -k_{1} y_{\infty}(t-X)+k_{2} y_{\infty}(t)+k_{3} R_{\infty}(t), \\
& 0 \leq t \leq \alpha \\
y_{\infty}(0)= & 0, \quad-X \leq t \leq 0 .
\end{aligned}
$$

An equivalent description obtained after some manipulation is

$$
\begin{aligned}
y_{\infty}(t)= & -\frac{k_{1}}{1-k_{2}} y_{\infty}(t-X)+\frac{k_{3}}{1-k_{2}} R_{\infty}(t), \\
& 0 \leq t \leq \alpha \\
y_{\infty}(0)= & 0, \quad-X \leq t \leq 0 .
\end{aligned}
$$

The limit profile for processes described by (1) and (3) has the same dynamics but a modified initial condition.

A somewhat surprising fact here is that asymptotic stability does not guarantee that the limit profile dynamics are "acceptable" in the along the pass direction. With a choice of $k_{2}$ for asymptotic stability, i.e., $\left|k_{2}\right|<1$, the (unconstrained by asymptotic stability) value of $k_{1}$ could cause unacceptable limit profile dynamics over $0 \leq t \leq \alpha$. For example, with $k_{1}=k_{3}=1$ and $k_{2}=0.8$ the resulting limit profile is described by

$$
\begin{aligned}
& y_{\infty}(t)=-5 y_{\infty}(t-X)+5 R_{\infty}(t) \\
& y_{\infty}(t)=0, \quad-X \leq t \leq 0
\end{aligned}
$$

and is easily seen to contain oscillations that increase in amplitude as $t$ ranges over the pass length $[0, \alpha]$.

For cases where this feature is not acceptable, the stronger concept of stability along the pass must be used (again, see [6] for the general theory). In effect, for the abstract model $S$, this requires that (4) holds uniformly with respect to the pass length $\alpha$. One of several equivalent statements of this property is the requirement that there exist finite real scalars $M_{\infty}>0$ and $\lambda_{\infty} \in(0,1)$, which are independent of $\alpha$ and satisfy

$$
\left\|L_{\alpha}^{k}\right\| \leq M_{\infty} \lambda_{\infty}^{k} \quad \forall \alpha>0 \quad \forall k \geq 0 .
$$


This property holds if, and only if

$$
r_{\infty}:=\sup _{\alpha>0} r\left(L_{\alpha}\right)<1
$$

and

$$
M_{0}:=\sup _{\alpha>0} \sup _{|\eta| \geq \lambda}\left\|\left(\eta I-L_{\alpha}\right)^{-1}\right\|<\infty
$$

for some real number $\lambda \in\left(r_{\infty}, 1\right)$.

The first condition here is simply the requirement that asymptotic stability holds for all possible values of the pass length $\alpha$ and not just the value of the particular example under consideration. Also, from the proof of Theorem 1 , it follows immediately that this condition holds if, and only if, $\left|k_{2}\right|<1$. Overall, we have the following result.

Theorem 2: The linear repetitive process $S$ generated by a process modeled by (1) and (2) is stable along the pass if, and only if

$$
\left|k_{1}\right|<1-\left|k_{2}\right|
$$

Proof: Given the requirement that $\left|k_{2}\right|<1$, it remains to interpret (8) for this case. To do this, we construct conditions for the boundedness of the solutions of (6) for all $|\eta| \geq \lambda$ and some scalar in the range $\left|k_{2}\right|<\lambda<1$. In particular, using the first two equations in the proof of Theorem 1, (6) reduces to the relation

$$
y(t)=-\frac{\eta k_{1}}{\eta-k_{2}} y(t-X)+\frac{k_{1} x_{0}(t-X)+x_{0}(t)}{\eta-k_{2}} .
$$

Hence, (8) holds if and only if

$$
\sup _{|\eta| \geq \lambda}\left|\frac{-\eta k_{1}}{\eta-k_{2}}\right|<1
$$

or, on using elementary graphical arguments,

$$
\left|k_{1}\right|<1-\left|k_{2}\right| \text {. }
$$

Finally, if this last condition holds then asymptotic stability, i.e., $\left|k_{2}\right|<1$, is an immediate consequence and hence we need only consider it in checking for stability along the pass.

In the case of processes described by (1) and (3), the necessary and sufficient conditions are $r(K)<1$ and $\left|k_{1}\right|<1-\left|k_{2}\right|$. The proof follows indentical steps to that for the case of processes described by (1) and (2) and hence the details are omitted here. Of critical importance is the fact that the structure of the pass initial conditions alone determines asymptotic stability and hence stability along the pass (the former is always necessary for the latter). For the numerical example given earlier with $k_{1}=k_{3}=1$ and $k_{2}=0.8$, asymptotic stability holds but not stability along the pass, and only in the case when the pass initial conditions are of the form (2). Next, we introduce the classical approach to stability analysis of the linear repetitive processes considered here and then the links and restrictions of this approach versus that given above are detailed.

The classical linear systems based approach to the stability analysis of (physically relevant) linear repetitive processes was first developed in [2]. In effect, this approach completely ignores the pass initial conditions and converts the underlying dynamics into those of an infinite length (or duration) single pass process by using the concept of the "total distance traversed" from $k=0$ and $t=0$. In particular, consider "position" $t$ on pass $k+1$. Then, the single variable $v=k \alpha+t$ is termed the "total distance traversed" relative to $k=0, t=0$. Conceptually, this can be visualized as joining the start of each new pass to the end point of the previous pass.

Using this concept, a repetitive process variable, say $y_{k}(t)$, is expressed as a function of $v$ defined on $[0, \infty]$ and (1) becomes

$$
y(v)=-k_{1} y(v-X)+k_{2} y(v-\alpha)+k_{3} R(v) .
$$

Now the repetitive process dynamics are said to be stable provided (10) is stable in the 1-D linear systems sense. Applying the Laplace transform to (10), the condition for stability in this sense is that

$$
p(s):=1+k_{1} e^{-s X}-k_{2} e^{-s \alpha} \neq 0, \quad \text { Re } s \geq 0
$$

i.e., all roots of the "characteristic equation" $p(s)$ lie in the open left half-plane.

Note again that in this approach to stability, the pass initial conditions are completely ignored. Also in the case of processes described by (1) and (2), stability in this sense depends on both $k_{1}$ and $k_{2}$ whereas asymptotic stability (Theorem 1) from the abstract model based theory only depends on $k_{2}$. Hence, if there is to be any link between these two approaches to stability analysis it must involve stability along the pass. This is investigated next by considering (11) as $\alpha \rightarrow \infty$. In particular, it will be shown that (9) — stability along the pass—is both necessary and sufficient for all roots of (11) to have strictly negative real parts for all possible choices of the pass length $\alpha$.

First suppose that (9) holds then

$$
\left|1+k_{1} e^{-s X}\right| \geq 1-\left|k_{1}\right|>\left|k_{2}\right| \geq\left|k_{2} e^{-s \alpha}\right|
$$

for all $\operatorname{Re} s \geq 0$ and $\alpha>0$, i.e., $p(s) \neq 0$ for Re $s \geq 0$ and $\alpha>0$. Conversely, if $p(s) \neq 0$ for Re $s \geq 0$ and $\alpha>0$ let $q$ be a real positive parameter and set

$$
s= \begin{cases}\frac{q+i \pi}{X}, & k_{1}>0 \\ \frac{q+i 2 \pi}{X}, & k_{1}<0 .\end{cases}
$$

Suppose also that $\alpha$ is an even positive integer multiple of $X$ if $k_{2}$ is positive, an odd integer multiple of $X$ if $k_{1}>0$ and $k_{2}<0$, and an odd integer multiple of $X / 2$ if both $k_{1}<0$ and $k_{2}<0$. Then, it follows that

$$
p(s)=1-\left|k_{1}\right| e^{-q}-\left|k_{2}\right| e^{-\operatorname{Re}(s \alpha)} \neq 0, \quad q \geq 0
$$

from which (9) - the condition for stability along the pass of processes described by (1) and (2)-follows immediately.

The key point to note here is that, since the pass initial conditions are ignored, (11) is the stability condition under the classical analysis for processes described by (1) under all possible choices of the pass initial conditions. Hence, unless these are actually zero this approach will lead to incorrect conclusions re process stability. If, however, the pass initial conditions are indeed zero, then, this approach gives the correct result for stability along the pass, the stability property which will be most often required in applications. This, in turn, means that classical tools can be used to design control schemes for such linear repetitive processes. 


\section{CONCLUSION}

This paper has considered two approaches to the stability analysis of linear repetitive processes described by a delay-difference equation-a model which arises in certain applications areas. This has shown that the approach based on the abstract model in a Banach space setting is more general than that based on classical 1-D linear systems stability analysis/tests. In particular, the former here allows proper account to be taken of the pass initial conditions which alone can cause instability in these processes. If, however, the pass initial conditions are zero then these stability conditions produce exactly the same condition for the property known as stability along the pass which is what will be required in many cases. This, in turn, releases classical design techniques for use in such cases.

The analysis of this paper can, in principle, be extended to other cases. In particular, it can be extended to differential and discrete linear repetitive processes which arise in a number of applications areas, such as ILC and the optimal control problem solution algorithms. Which route to use would then be determined by the eventual end goals of the analysis (e.g., stability tests only or stability tests plus controller design) but in all cases the key message is that the structure of the boundary conditions alone and, in particular, the pass initial conditions determine the stability of these processes and hence they must be appropriately modeled.

\section{REFERENCES}

[1] S. E. Benton, "Analysis and control of linear repetitive processes," Ph.D. dissertation, School Electronics Comp. Sci., Univ. Southampton, Southampton, U.K., 2000.

[2] J. B. Edwards, "Stability problems in the control of multipass processes," Proc. Inst. Elect. Eng., vol. 121 , no. 11, pp. 1425-1431, 1974.

[3] K. Galkowski and J. Wood, Eds., Multidimensional Signals, Circuits and Systems. London, U.K.: Taylor \& Francis, 2001.

[4] D. H. Owens, N. Amann, E. Rogers, and M. C. French, "Analysis of iterative learning control schemes-A 2-D systems/repetitive processes approach," Multidim. Syst. Signal Processing, vol. 11, no. 1/2, pp. 125-177, 2000.

[5] P. D. Roberts, "Two-dimensional analysis of an iterative nonlinear optimal control algorithm," IEEE Trans. Circuits Syst. I, vol. 49, pp. 872-878, June 2002.

[6] E. Rogers and D. H. Owens, Stability Analysis for Linear Repetitive Processes. Berlin, Germany: Springer-Verlag, 1992, vol. 175. 